The erosion of nongambling spheres by smartphone gambling: A qualitative study on workplace and domestic disordered gambling
Mobile Media \& Communication

(C) The Author(s) 2020 Article reuse guidelines: sagepub.com/journals-permissions DOI: $10.1177 / 2050157920952127$ journals.sagepub.com/home/mmc

(S)AGE

\author{
Hibai Lopez-Gonzalez \\ Department of Psychiatry, University Hospital of Bellvitge-IDIBELL, Spain; Department of Library, \\ Information Science and Communication, University of Barcelona, Spain
}

\title{
Susana Jiménez-Murcia
}

Department of Psychiatry, University Hospital of Bellvitge-IDIBELL, Spain; Ciber Fisiopatologia Obesidad y Nutrición (CIBERobn), Instituto Salud Carlos III, Spain; Department of Clinical Sciences, University of Barcelona, Spain

\section{Mark D Griffiths}

International Gaming Research Unit, Nottingham Trent University, UK

\begin{abstract}
The potential dangers of internet-based gambling as compared to more traditional landbased gambling have been increasingly investigated over the past decade. The general consensus appears to be that, while internet gambling might not be a more dangerous medium for gambling per se, the 24/7 availability it generates for problem gamblers, is. However, since smartphones have become the most used way to gamble online, internet gambling must be further subcategorised regarding the device of access. This study examines the issue by exploring the views of smartphone gamblers undergoing treatment for gambling disorder in focus group settings $(\mathrm{N}=35)$. Utilising thematic analysis, the paper shows that smartphone gambling has colonised spaces previously regarded as nongambling spheres. The workplace, especially in male-dominated contexts, emerged as an accommodator and stimulator of gambling behaviour, raising issues of productivity rather than criminality. Domestic gambling was mostly characterised by an invasion of bathroom and bedtime spheres of intimacy. The study examines the implications for prevention and treatment, focusing on the minimisation of exposure to gambling stimuli, the erosion of intimacy that recovering gamblers must endure, and the necessity to embrace a broader definition of gambling-related harm.
\end{abstract}

\section{Keywords}

Smartphone gambling, mobile phone gambling, workplace gambling, intimacy, sports betting, gambling disorder, domestic gambling 


\section{Introduction}

Nationwide representative data from multiple territories, as well as industry-level and academic reports, largely testify to the global growth of the online gambling market, sometimes to the detriment of land-based modes of gambling (e.g., Gray et al., 2015; LaPlante et al., 2008; Myllymaa, 2017). Logically, early accounts about online gambling assumed this was carried out via desktop computers and laptops, as mobile technologies were not sufficiently developed at the time (Griffiths \& Parke, 2002). However, as mobile devices became integral parts of everyday life and their access to reliable mobile networks more affordable, smartphones rapidly evolved into the method of preference to gamble online. For instance, in the UK, smartphones are the most used device for online gambling, increasing from $23 \%$ in 2015 to $50 \%$ in 2019 . On the contrary, gambling via laptops decreased from $61 \%$ to $38 \%$ in the same period. In particular, for those between the ages of 18 and 24 years who gambled online, $74 \%$ used their smartphone to do so (Gambling Commission, 2020).

These developments have arguably transformed smartphone gambling into the epitome of online gambling, and with that, public health concerns about the expansion of commercial gambling have conflated with analogous concerns about the excessive penetration of smartphones in everyday activities. The most apparent causes for unease can be probably associated to the constant accessibility and 24/7 availability of online gambling. This has made gambling evolve from a discontinuous form of consumption (e.g., subject to opening hours of casinos and physical proximity) into a continuous one that allows uninterrupted, faster gambling (Parke \& Parke, 2019).

Given these constraints and affordances of mobile, internet-based devices, online gambling has been generally considered a gambling form with greater addictive potential. For example, in a sample of 800 regular gamblers from Québec, online gamblers exhibited worse work productivity, more sleep problems, and their physical health more affected. Mixed gamblers (i.e., gambling both online and land-based) showed bigger problems, but when comparing pure online versus pure offline gamblers, those in the online condition had greater impairment and negative consequences (Papineau et al., 2018). In another study with Spanish adolescents aged 14-18 years it was found that among the non-problem gambling group, $90 \%$ of participants were land-based and only $9 \%$ were online or mixed. However, very notably, as gambling severity grew, the 
percentage of mixed gamblers increased to 33\% (González-Roz et al., 2017). Similarly, in a study with Portuguese gamblers, online gamblers spent more hours gambling, and were eight years younger than offline gamblers (Hubert \& Griffiths, 2018).

Nevertheless, the authors in the previous study pointed out that online gambling might not be a more problematic medium per se. The real issue being that it gives continuous access to those who are already experiencing gambling problems. A large-scale French study $(\mathrm{N}=9910)$ provides support to this interpretation. Although online gambling was a predictor of gambling disorder and money spent gambling, the association disappeared when controlling for time spent gambling and number of gambling types (Baggio et al., 2017). Two other studies using largescale nationally representative data from British gamblers also demonstrate that while the prevalence of problem gambling was significantly higher among those that have used the internet to gamble $(5 \%)$ compared to those who had not $(0.5 \%)$, most online gamblers also gamble offline (Wardle et al., 2011). All in all, the defendants of this perspective contend that online gambling might not be deleterious on its own, but more likely gamblers experiencing problems resort more often to all sorts of gambling modes in order to increase their consumption when solely landbased gambling products restrict too much the kind of gambling availability they require (Wardle et al., 2011). This view would also explain why mixed gamblers appear to be the ones suffering more severe gambling problems.

Notwithstanding these contrasting views, it appears that online gamblers are systematically overrepresented among problem gambling groups as opposed to landbased gamblers, something even more visible among smartphone gamblers (Braverman et al., 2013). However, the prevalence data on how gamblers engage in gambling fails to capture the unique underlying features of online gambling, and cannot properly account for the distinctively addictive-inducing characteristics of online gambling products.

\section{Smartphones as distinctive gambling devices}

There is limited evidence about the effect of smartphone use to gamble versus other internet-based methods. In a survey study with 4,800 Australian respondents, and after controlling for sociodemographic covariates, online gamblers who used supplementary devices to gamble versus PC-based gamblers were found to be twice as likely to be problem gamblers (Gainsbury et al., 2016). Naturally, younger individuals are more likely 
than their older peers to engage in smartphone gambling (Zhao et al., 2018). However, although younger gamblers are at greater risks of experiencing psychological distress and gambling-related harm than more adult gamblers, this does not necessarily mean smartphone gambling is more problematic or addictive. It could simply be interpreted as an outcome of age-related behaviour and that any platform or device favoured by adolescents would automatically become associated with more problematic indicators (Gainsbury et al., 2019).

Smartphone gambling has been conceptualised by some authors as anti-social, antifamily, and isolationist (Drennan et al., 2018). However, more technically speaking, potentially the most adverse interaction between gambling and smartphones is the intermittent, sporadic, interspersed bouts of engagement that users have with their mobile devices and how this resonates with reinforcement programs present in gambling products. James and colleagues (2017) refer to such intermittent smartphone interaction by using the term 'snacking'. Smartphones are used for short but frequent bouts (as opposed to long continued periods of time), in line with data from industry reports estimating that the average smartphone user interacts with their device 2,617 times per day on average (Winnick \& Zolna, 2016). The claim here is that many sporadic short interactions with the phone make humans learn the behaviour; if gambling products punctuate these interactions with intermittent prizes (i.e., positive reinforcement schedules), the human brain acquires the habit and demands more of the same. Paradoxically, the longer the period between prizes (i.e., latency), the faster the acquisition of the learned behaviour, meaning that random ratio reinforcement schedules in smartphone gambling are particularly good at solidifying this learning path (James et al., 2017). It can be argued that product designers involved in gambling/gaming as well as smartphone products fine-tune the human-device interaction to elicit human responses that better align with their commercial goals, maximising the reinforcement ability of their device. Importantly, this can be achieved even in the event of unsuccessful gambling outcomes, which-adequately designed-prove to be very powerful behaviour reinforcers (James et al., 2019).

\section{Smartphone gambling invading non-gambling spheres}

The end of temporal constraints that online gambling brought about (i.e., individuals could gamble $24 / 7$ if they so wished as opposed to only gambling when gambling venues 
were open) was extended to its spatial constraints with the advent of smartphone gambling (i.e., individuals could gamble anywhere with a mobile phone as opposed to only being able to gamble via a computer at their home or workplace). In short, smartphone gambling can happen virtually anytime and anywhere and one of the consequences is its penetration on non-gambling spheres. The erosion of privacy versus public space boundaries and the loss of privacy and intimacy-public-in-private spaces (Sheller \& Urry, 2003) — that other smartphone uses entail (e.g., selfies or social media posting) works slightly different when it comes to smartphone gambling. At home, 'domestic gambling' has introduced the gambling device into the bedroom and has colonised bedtime rituals, arguably reducing sleeping time amongst those excessively engaged with the online activities (including gambling) (Alimoradi et al., 2019). Bathroom rituals might have also been similarly disrupted by domestic gambling. However, no previous study has ever explored the colonisation of intimate spheres that domestic smartphone gambling has likely caused, something this study aims to remedy.

More evidence is available for the penetration of online gambling in the workplace. From a historical point of view, office sweepstake has been a traditional feature of some work environments and often considered a benign form of leisure that promotes team-building (Griffiths, 2009). In some male-dominated work cultures, 70\% of employees report having participated in office betting pools (Vandewater, 2013). In this traditional context, problems associated with workplace gambling do not derive from the fact that individuals gamble at work per se, but from problem gambling behaviour from outside work that eventually has an impact on work. These problems materialise in being late for work, leaving early, abusing vacation and leave time, neglect of work duties, irritability at work, lack of attention and focus, occupational hazards, and ultimately, embezzlement and fencing stolen property to finance gambling (Griffiths, 2009; Paul \& Townsend, 1998). Indeed, studies from multiple countries have estimated that about $22-37 \%$ of problem gamblers have stolen or embezzled money from work (Binde, 2016). However, traditionally, the majority of jobs were physically incompatible with workplace gambling because employees were supervised and the concept merely referred to the occupational consequences of free time gambling.

The advent of the internet created many gambling-compatible workplaces in which workers could access gambling sites from their desktop computers. Here, the focus shifted 
from embezzlement-related issues to productivity issues, with employers preoccupied about the use of new technologies at work for non-work-related activities (Fox et al., 2003; Griffiths, 2009), and which prompted the first studies to examine the problems generated by those gambling at work (Nower, 2003). However, there is a shortage of empirical evidence regarding (i) the effects of workplace gambling since the popularisation of smartphone gambling, and (ii) how specific work cultures combined with smartphone technologies can trigger gambling problems (something previously observed in gambling venue employees who are constantly exposed to gambling stimuli) (Hing \& Gainsbury, 2013).

\section{This study}

The present study aims to understand the impact of the penetration of smartphone gambling in non-gambling spheres of everyday life that were previously off-limits for gambling products. The underlying assumption is that smartphone gambling has expanded beyond traditional gambling-compatible temporal and spatial opportunities, with observable effects on how intimate or private spheres such as domestic spaces have been colonised, causing disruptions in the way gamblers interact with other individuals in such spaces. Also, workplace gambling is examined as a space with limited access to gambling in the past that now has seen its availability increased, in which smartphone gambling might generate conflicts. With this aim in mind, data from the experiences of gamblers undergoing treatment for gambling disorder were collected because they were the more likely candidates to have seen all the spheres of their life compromised by smartphone gambling.

\section{Method}

\section{Participants and procedure}

A qualitative approach based on focus group interviews was favoured to analyse the verbal accounts of people with gambling disorder in recovery. A sample of 40 participants was recruited from different Spanish regions. In total, the research team carried out six focus groups in five cities from March to June 2019, in the following chronological order: Vigo $(n=8)$, A Coruña $(n=6)$, Oviedo $(n=16)$, Sevilla $(n=4$ and $n=3)$, and Leganés $(n=3)$. The mean age of the participants was 29.9 years-old $(\mathrm{SD}=7.04)$, and their mean age for the onset of gambling was 21.6 years $(\mathrm{SD}=6.5)$, which meant that, on average, they had been gambling for about eight years before seeking professional help. The vast majority 
of participants were males (39 men and one woman). Their preferred gambling type was also unevenly distributed: sports betting $(n=26 ; 63 \%)$, online roulette $(n=2 ; 5 \%)$, online slot machines $(n=2 ; 5 \%)$, online poker $(n=2 ; 5 \%)$, online tombola $(n=1 ; 2.5 \%)$, gaming (these were later excluded, $n=2 ; 5 \%$ ), and five did not disclose it (although they had previously stated that it was gambling online). In terms of education, $34 \%$ had vocational/technical training, 29\% had university level education, $22 \%$ graduated in high school, and 5\% did not finish secondary education. Four participants did not respond. Detailed data for each participant is available for downloading (Supplementary Table 1).

The participants were a non-probabilistic convenience sample recruited via collaborating associations of the Spanish Federation of Rehabilitated Gamblers (Federacion Espanola de Jugadores de Azar Rehabilitados [FEJAR]), the largest national provider of treatment for gambling disorder in the country. Each association was contacted asking for participants who fulfilled four main criteria: (i) being adults ( $\geq 18$ years); (ii) having been diagnosed with gambling disorder and currently undergoing treatment for it, (iii) online gambling being their predominant mode of gambling, and (iv) the smartphone being their preferred way of engaging in online gambling. This last criterion was not fulfilled by five participants (who initially reported otherwise or due to a communication error by the association. This resulted in a final sample of 35 participants (all male). All individuals undergoing treatment in the associations and who met the inclusion criteria were given the opportunity to participate, meaning that resulting participants self-selected for the study. The research team is unaware of the reasons of those who decided not to participate.

The associations that were able to recruit at least three individuals willing to participate in the study were contacted by email or telephone to arrange a focus group in their facilities. The first author of the study (a male $\mathrm{PhD}$ in Communication) travelled to the site and conducted all the interviews, informing participants of his background and published experience in running focus groups, and the goals of the study. The researcher was not previously acquainted with the participants and reported to them no bias that could influence the development of the study. All focus groups were audiotaped and then transcribed by another team member and no field notes were taken. Only the leading author and the participants were present in the room except in one case (Leganés; a female 
psychologist was in the room). Participants did not receive transcripts and were not contacted for comment or feedback on the findings.

\section{Ethics}

The design of this study was reviewed and approved by the ethics committee of the first author's institution (Ref: ETK-38/18-19). All participants signed consent forms and were informed about the concept of the study and their rights to abandon at any time without any repercussions to their treatment. Also, they were reassured about the anonymity of their responses and inform about the research members that will have access to the recording. Participants were compensated for their time with a gift of earphones $(\sim € 8$ 10). The study adhered to the guidelines of the EQUATOR Network to enhance the quality and transparency of health research. In particular, it follows the Consolidated Criteria for Reporting Qualitative Research (COREQ) and complies with the 32-item checklist for interviews and focus groups (Tong et al., 2007). (See Supplementary Table 2)

\section{Data collection and analytical approach}

The focus groups had an average duration of 73 minutes (range $=45-97$ minutes). The initial script was the same for every group and contained six blocks. These blocks covered (i) differences between online and offline gambling, (ii) other online-related problematic behaviours, (iii) gambling-related socialisation using online tools, (iv) perceived obstacles to seek for help for online gamblers, (v) differences in group therapy between offline and online gamblers and challenges for relapse prevention in online gambling, and (vi) gender issues in online gambling (see Supplementary File 3 for a detailed interview guide).

This script was used merely as a starting point to boost the conversation and as a guidance, but the researcher on site was flexible within each block to pursue serendipitous findings and aspects that participants spontaneously considered significant. In fact, the specific topics of the present paper (i.e., smartphone gambling, erosion of intimacy, workplace gambling) were not predetermined in the script, and only emerged during preliminary data analysis of mostly blocks 1,2 , and 3 . These preliminary explorations were conducted within days after each focus group took place, emphasising the iterative, circular process of 'data-gathering-analysis-data-gathering-analysis' in many qualitative investigations 
(see Hartley, 2004; Kohlbacher, 2006). For this reason, excerpts from the latter focus groups in Sevilla and Leganés are more frequently cited, as the researcher was already trying to elaborate further on the tentative finding that articulates the present paper.

Data were analysed using NVivo 11 for Mac. Only one coder uploaded the data into the software and conducted the preliminary coding of nodes, but two more coders independently reviewed the totality of the material with an analogue coding and participated in the successive analysis and refinement of codes, as well as the writing of the final report for the organisation that commissioned the study. The focus groups were conducted in Spanish, and the materials were analysed and coded in this language. Some selected quotes were translated into English for the purposes of providing exemplars in the present paper - a process supervised by a native English speaker.

The authors examined the data utilizing thematic analysis and an inductive approach. This method is particularly useful for uncovering latent factors in participants' stories, identifying regularities in terms of thematic nodes, and creating over-arching narratives that make sense of the content of the discussion in the focus groups (Braun \& Clarke, 2006). No aprioristic theoretical framework was favoured to enable any possible interpretation consistent with the data. Consistent with Saldaña's coding recommendations (2009), the coding process had three main phases and was designed to not replicate the blocks outlined in the initial interview script: (i) holistic coding, by which the main themes were first identified and categorised as broadly as possible, (ii) in parallel to holistic coding, in vivo coding, which tagged word-by-word pieces of discourse by participants that were particularly illustrative of a concept, and (iii) structural coding, which is a second cycle coding, regrouped the initial coding and merged and transformed the nodes into a new classification. This process was iterative and facilitated researchers to familiarise themselves with the data. The coders frequently shared their preliminary results with the rest of the coding team. All three participated in the holistic, in vivo, and structural coding.

The preliminary holistic coding identified 1023 code references and grouper the content in 58 nodes. These nodes were later simplified and merged into 23 new nodes during the structural coding, which corresponded to four main categories: (i) differences between online and offline gambling (ten nodes), (ii) problems in the online gambler protection 
(four nodes), (iii) the role of smartphones and social networks in online gambling (five nodes), and (iv) chance and skill balance in online gambling (four nodes; a detailed list of all the nodes and the number of times each node was referenced is available in Supplementary Table 4). The research team thought that this classification did not exhaust the analysis and operated a cross-sectional examination of all the material. This secondcycle coding was no longer inductive because it scrutinised deductively the interviews looking for connections between smartphone gambling and its nodes. The resulting data were finally organised in two new categories (workplace gambling and domestic gambling) all responding to a single all-encompassing narrative theorised to be the colonisation of non-gambling spaces by smartphone gambling'. These categories are developed in detail in the next section. For an approximate understanding about how many participants endorsed each theme, the terms 'most' ( $80 \%$ of the participants or higher); 'many' (50-79\%); 'some' (20-49\%), and 'a few' (19\% or below) are used.

\section{Results}

Two main themes were identified in the analysis: workplace gambling and domestic gambling. The following section details the main subthemes, which are visually summarised at the end (Figure 1).

\section{Workplace gambling as accommodator and stimulator}

Most of the interviewees reported resorting to their smartphones to gamble while working, using different strategies to adapt to and exploit the possibilities their job offers to gamble. In general, two main themes emerged from the analysis concerning smartphone gambling at work: the workplace as gambling accommodator and the workplace as gambling stimulator.

On the one hand, workplaces that accommodate gambling are those that because of their structural characteristics are particularly compatible with gambling behaviour. Examining the job descriptions of the participants, a number of patterns arise: (i) repetitive and tedious jobs that can be carried out mechanically with low concentration (e.g., assembly line worker), (ii) jobs involving long waiting periods (e.g., ambulance support technician) in which mobile use becomes common in an effort to fight boredom, and (iii) working nightshifts, which allows for less supervision and sometimes also recurrent periods of inactivity (e.g., cleaners, security guards, customer service jobs at 
call centres). The term 'gambling accommodator' acknowledges the fact that most workspaces do not preclude workers from using their phones but indicates the particularly easy access that some jobs have in contrast to others, such as this one: "I've been in the nightshift in my factory for eight years now. 12 hours every day. At 9pm the dayshift leaves, and I connect to the poker tournament. Sometimes I get kicked out at lam, sometimes I'm up to the last table at 6am" (P11, 31 years).

On the other hand, some workplaces go from accommodating gambling to stimulating it. In this regard, three main subthemes were identified. First, male-dominated work environments with few female workmates appeared to be predominant among participants, wherein sports betting was perceived as a natural conversation driver. Many participants perceived that gambling in their workspace is widely practiced and accepted, which they felt clouded their understanding of gambling as a potentially pernicious activity. Such circumstances were closely linked to the second subtheme, which revolved around the idea of gambling communities in the workspace. Some participants reported having WhatsApp groups with workmates that were used for the sole purpose of discussing sports bets. These groups were sometimes used to pool money for a specific bet, the procedure being that one worker fronted the money, placed the bet on behalf of the group members, and shared the picture of the receipt with them. The community gambling dynamic had some damaging outcomes for some individuals. One participant abstinent from gambling, who was on sick leave at the time of the interviews, mentioned that the betting discussions with his colleagues were what he missed the most from work.

A few participants reported using their workplace gambling communities to enable their gambling in moments where they were not able to engage in the activity. For example, one gambler mentioned being in family events where using his phone to bet would not be appropriate and how he resorted to their WhatsApp group to request workmates to place bets on his behalf. These groups were also a source of distress for gamblers in recovery, since often they have to conflate keeping in secret their gambling condition with stopping interacting in gambling groups for no apparent reason, something that prompted participants to complain about the peer pressure they were subject to. One participant explains it this way: "I used to chip in with $€ 2$. And now they tell me: what's wrong with you now? And I tell them: not interested, I'd rather save the $€ 2$ for something else, we always lose" (P40, 35 years). 
The third subtheme about the workplace as gambling stimulator was a combination of the first two. A male-dominated work atmosphere combined with a work gambling community produced pernicious environments for those who wanted to cease or cut down their gambling habit. Some participants referred to network dynamics by which colleagues showed them mobile screen captures of large bets or shared such captures via work WhatsApp groups in order to brag about their audacity and gambling knowledge. One participant disclosed that he began gambling when joining his current position: "I started as everybody does, at work someone came and said 'I've won €300', and I signed in for the first time with a bonus offer, and won some money in the beginning, but lost all of it and plenty more later" (P39, 31 years). The use of the terms 'everybody does' connotes a naturalisation of male workplace cultures as instigators of gambling behaviour. Participants also referred to them being the ones who shared winning betting receipts while ignoring lost bets. This same participant (P39) also recalled gloating over a $€ 1,500$ bet win visible on his smartphone screen.

\section{Gambling-tolerant versus gambling-intolerant workplaces}

A particularly problematic work environment where male culture meets gambling culture meets sport was the locker room of a professional team. One of the participants was a former professional athlete in a popular team sport in Spain. He recounted how he managed to continue his betting during games: "When I was competing, during halftime breaks, I caught my phone, waited for somebody else to leave the locker room, and checked the game, how my horse was doing...I just checked because I had no time for anything else" (P5, 33 years). This participant shared how the gambling culture in his professional environment worked. He admitted signing several documents that precluded him from betting but acknowledged that everybody else bet. Also, that he bet on his own competition and that players usually called each other before games to enquire about the latest news (e.g., who is injured, who is in good shape, etc.) so they could make informed decisions about their bets. However, he was reluctant to entertain the idea that this might constitute some sort of match-fixing scheme.

Work environments that accommodate and/or stimulate gambling differed significantly from works where gambling was not tolerated. One particularly illustrative case came from this participant: "I'm a schoolteacher, and I was with the kids and gambling, which 
is really embarrassing to say...gambling on my phone when I was teaching in class" (P10, 59 years). In this extreme case, the gambling activity constituted a social transgression that increased the gamblers' feelings of guilt, who knew about the inappropriateness of his behaviour while acknowledging his inability to refrain.

\section{Novel workplace hazards}

A theme typically associated with workplace gambling but absent from the analysis was embezzlement, which was not identified as a theme here except in one case. Coincidentally, this participant was a former betting shop manager who admitted to using the internal network system of his shop to place bets (something that is strictly forbidden) without actually providing the money, in the hope that winning bets would allow him to compensate for the wagered amount and, on top of that, make a profit.

In general, smartphone gambling in the workplace appeared to be more associated with productivity issues rather than criminal acts. A major subtheme that stood out in this regard was new workplace hazards that departed from traditional gambling hazards. Three participants mentioned having jobs that involved driving or handling heavy machinery, with two of them reporting smartphone gambling-related accidents or nearaccident experiences: "When I was driving my truck I bet on a game... and then spent three hours checking the progress of the game...it gave me a fright several times" (P7, 32 years). Participant 40, who is a regular line bus driver, recounted a similar story but felt ambivalent about the implications of his behaviour, exhibiting resistance to admitting that he put passengers in danger because of his smartphone gambling:

I used my ten-minute breaks to smoke and check my bets. The worst part was when I was driving and the alert went off [phone notification], and you wondered who scored or what happened...always paying attention to that... [Researcher: I imagine driving and checking must have been dangerous] No, I mean, I always had it under control. I mean, gambling was never under control but, for example, I never checked the phone while driving, I only checked during stops (P40, 35 years).

This idiosyncratic aspect of smartphone gambling illustrates the constant inhibitory control required by gamblers during their everyday tasks including work. It highlights the 
pervasive nature of smartphone gambling, which cannot solely be understood as the time actually spent betting on the device but the aggregate of betting plus permanent update checking.

Another novel workplace hazard was associated with commuting. Those who commuted to work by public transportation often engaged in smartphone gambling during their journey. A few participants described-especially during the early stages of their addiction - feeling ashamed if somebody on the transport caught them gambling but reported that as their condition worsened, the shame vanished and they only wanted to be left undisturbed.

\section{Domestic gambling}

Most of the participants referred situations in which they gambled on their smartphones in intimate spaces at home such as the bathroom and, for those gamblers who lived and slept with their partner, their shared bed. During the focus groups, the researcher did not lead any participants to talk about their gambling in such spaces, and this was not part of the interview script. However, as a theme about the colonisation of intimate spaces by smartphone gambling emerged during the preliminary examination of the transcripts, at some point during data collection, follow-up questions were included to further scrutinise participants about it.

\section{Bathroom gambling}

Many participants typically referred to episodes of 'bathroom gambling' due to the protection and intimacy that this space procured them. It happened both in household and work situations, to avoid the eyes of their partner, family and/or line manager. Some of the participants (e.g., P14, 26 years) who was cohabiting with a partner mentioned pretending to be sick - typically about something he had eaten - to have an excuse to spend long periods of time in the toilet with the door locked. Another participant (P35, 25 years) said that he constantly switched from laptop to smartphone and vice versa but preferred his smartphone because this way he did not have to stop gambling in the bathroom. Bathroom and workplace gambling intersected when participants recounted using their breaks at work to lock in a toilet stall with their smartphone to gamble. 
There were also some participants who acknowledged that the penetration of smartphone gambling in their most intimate spaces during their gambling activity had implications now that they were in recovery. In the following excerpt, two participants discuss the loss of personal space one of them had experienced since starting therapy:

P38 (44 years): "I have gone many times to the toilet, pretending I was going to take a $s^{* * *}$, but it was actually to check a $f^{* * * * * *}$ bet. I used to sit on the toilet lid with the phone on my lap...but now every day is open house day, closed doors are not allowed, I know it sounds silly, but we [my wife and I] have talked to each other a lot...I'm having a shower and my wife is there, I mean...

P39 (31 years): Come on, having a shower...ok, but... [insinuates defecation] P38: That's the way it is now!

The process of regaining the trust and confidence of their partner and family sometimes entailed giving them access to moments of personal intimacy, which was not perceived by online gamblers to be necessarily true for land-based gamblers who shared group treatment with them. Therefore, the more areas their gambling was able to penetrate, the greater the transparency they would need to prove that their gambling was over. As smartphone gamblers were able to basically engage in gambling at any time in any place, they were required to demonstrate their abstinence in a wider range of situations. For some participants, this materialised in granting their partners and a few significant others frequent access to their smartphones, and in one extreme case, installing parental control software to supervise their online activity (this occurred in a marital relationship, not a parental one).

\section{Bedtime gambling}

Bedtime implications was also a theme brought up by some participants. Broadly speaking, online gamblers reported suffering sleep deprivation as a consequence of their overnight gambling. Three subthemes were identified that detailed this type of gambling: (i) gamblers that spent their night awake gambling (e.g., online poker tournament gamblers), (ii) gamblers that kept their phones in bed with them and gambled until they fell asleep, and (iii) gamblers that set up alarms to wake up in the middle of the night to check the outcome of a bet (e.g., online sport bettors who bet on American sports leagues). One participant did not see bedtime gambling as a sign of inability to pause his 
gambling and made sense of it by arguing that "online bingo at 3-4am is much higher" (P6, 33 years) [presumably meaning that prizes are larger or more likely at that time].

Those participants who slept with a partner were forced to lie about why they stayed out of bed, or got up, and had to conceal their gambling in bed. A few participants reported gambling with their phones under the sheets and the screen set to minimum brightness, in unorthodox positions to keep the device as far away from their partner as possible. Others simulated insomnia or stomach ache to justify getting up, not coming to bed, or staying in the bathroom.

Two participants reported that their partners pretended to be asleep to catch them gambling under the sheets, but even in those circumstances, they denied it. Smartphone use and smartphone gambling might look like identical behaviours from the viewpoint of an outside observer who is not familiarised with it and has no visual access to the display, a confusion that was not possible in traditional gambling. One participant acknowledged to emphatically deny his wife's accusation: "My wife: are you gambling? Me: No, no, no, I'm not, are you crazy? How am I gonna be gambling here?" (P40, 35 years). This behaviour might be interpreted as gaslighting.

Insert Figure 1 about here

\section{Discussion}

The present study examined the impact on domestic and workspace spheres of those who engaged in smartphone gambling and were at the time of the study receiving treatment for gambling disorder. The findings support and extend the main tenets of the transition from discontinuous to continuous forms of contemporary gambling proposed elsewhere (Dickerson \& O'Connor, 2006; Parke \& Parke, 2019). Although such transition was already observable since the early developments of internet-based gambling, the present study provides evidence about the extent to which smartphones have pushed gambling into a new, augmented definition of continuous behaviour. The internet markedly increased the availability of gambling products (Wardle et al., 2014) but as long as they remained confined to the boundaries of desktop computers and laptops, their accessibility was limited. Smartphones have not only traversed those remaining boundaries, but appear to have exploited the constant checking and notification patterns inherent to 
contemporary phone utilisation to virtually inundate any imaginable sphere of everyday life.

The previous point merits further elaboration. To date, evidence has repeatedly demonstrated that online gamblers tend to be of younger age as compared to land-based gamblers (e.g., Chóliz et al., 2019; Lloyd et al., 2010; Wardle et al., 2011). Online gamblers are also more prone than land-based gamblers to incur more rapid and higher debt, and to develop severe gambling disorder earlier (Estévez et al., 2017; Gainsbury et al., 2014; Mihaylova et al., 2013). Very notably, the implications of the findings from the present paper suggest that smartphone gambling might theoretically accelerate gambling disorder and debt processes. Traditional slot machine gamblers suffering with gambling disorder had to subtract from their daily gambling, at least, a number of compulsory resting periods due to inaccessibility to slot machines (e.g., night time, work, study, and/or family obligations, or commuting). Conversely, an online slot machine gambler who uses the smartphone to gamble can overcome those access limitations and (theoretically) occupy most, if not all, of the day gambling.

This provides a new interpretation to studies reporting for online gamblers a much faster evolution since the onset of gambling behaviour to the development of gambling disorder (Jiménez-Murcia et al., 2010), ratifying 'intensity' of gambling as a more accurate indicator than the number of 'years' spent gambling. This is further supported by evidence from experimental studies that point out how trial-based conditioning (i.e., how frequently gambling happens) is more relevant for intermittent ratio reinforcement (i.e., random prizes) than timing-based trials (i.e., how long the learning period was) (Bouton et al., 2014). Interestingly, trial-based conditioning might be closely related to the 'telescoping effect', the tendency of late-onset gamblers (typically women) to more rapidly progress their gambling disorder. Gambling type as well as non-strategic gambling have been proposed as determinants for telescoping (Grant et al., 2012) but other studies have not been able to replicate these results (Zakiniaeiz et al., 2017). The present study speculates that, perhaps more importantly than type, some structural characteristics of specific games which accelerate the trial-based conditioning are the key feature to explain telescoping. 
The present findings have severe implications for the treatment of the smartphone disordered gamblers. Since cognitive-behavioural therapy continues to be the 'gold standard' for gambling disorder treatment (Petry, 2016; Tolchard \& Battersby, 2013), the control of gambling stimuli is essential. Gamblers are expected to limit their exposure to gambling-related cues (e.g., walking by casinos, watching gambling advertisements, or entering establishments with slot machines) to minimise their craving and avoid a relapse. The pervasiveness of smartphone gambling and the stimuli associated with it make it much harder here to properly control the exposure as compared to traditional gambling, which could be achieved simply by eluding specific locations. As smartphone gambling had penetrated every space of the intimacy of these participants, they had to allow access to them in order to recover control over their gambling. Arguably, the culpability for having lied and failed to significant others might have fuelled the acceptance of such loss of privacy and enhanced scrutiny.

The gambling colonisation of intimate spaces is especially relevant considering that $95 \%$ of online gambling happens at home (Gambling Commission, 2020). The fact that the vast majority of online gambling is located within the household might have other less obvious implications. First, it might mean that gambling causes gamblers to neglect other family members at home, producing a less heathy home environment. Second, online gambling - even if done hiddenly - might be eventually be seen by children at home. A gene-environment interplay is assumed by most modern gambling models that try to account for the effect of family on problem gambling (Slutske, 2019). Children who see their parents gambling at home might be more likely to normalise gambling behaviour and engage themselves in gambling earlier in their lives. As such, the implications of the present study transcend the mere remit of gambling disorder, and are more deeply rooted in the gambling-related harm paradigm that considers problem gambling as a multifaceted construct that expands beyond the individual problem gambler (Browne et al., 2017).

In terms of workplace gambling, the present paper departs from previous studies concerning the effects on work of those gambling outside the workplace (Binde, 2016; Paul \& Townsend, 1998) and complements evidence from early studies about computer and telephone gambling at work (Griffiths, 2009; Nower, 2003). The present findings clearly situate smartphone gambling at work as facilitated and sometimes accelerated by 
work environments. The male-dominated work culture in which many of the participants in the present study appeared to be working naturalised gambling behaviours and helped to underestimate risks. As observed in the context of alcohol consumption in the workspace, the strongest predictor for drinking alcohol at work is the perceived workrelated drinking by others (Ames \& Grube, 1999). This speaks volumes about the importance of descriptive norms (i.e., what people think others are doing) and has already been theorised to have a significant influence in gambling contexts (Deans et al., 2017).

\section{Limitations}

The limitations in this study mainly involve the sample recruitment and analytical approach. Participants self-selected for the study given a number of inclusion/exclusion criteria which could have biased the final sample, although it is unclear whether those experiencing less problems at the moment would be more open to share or, on the contrary, those experiencing more severe problems would find a higher urgency. The focus group structure allowed for a more naturalistic setting to share views, especially in discussing behaviours that are to an extent social such as workplace gambling and sports betting. However, in contrast to individual interviews, participants in focus groups are more vulnerable to exhibiting social desirability biases (Bergen \& Labonté, 2019). Furthermore, the present study only included male online gamblers because no females meeting the inclusion criteria were located. Therefore, these results cannot be generalised to female online gamblers.

Another potential source of bias has to do with participants predominantly using their smartphones to bet solely on sports. This type of gambling has been contended before to be a growing concern in terms of public health impact, particularly when it is conducted in its most immediate and impulsive forms via in-play betting (LaPlante et al., 2008). In Spain, sports betting constitutes a big proportion of the online gambling market, and research from other jurisdictions shows that problems related to sports betting are much more common amongst those gambling online than land-based (Gainsbury et al., 2019). This is why it could be argued that sports betting is a convenient way to explore smartphone gambling, notably so because (i) gamblers need to simultaneously use a primary screen to watch the game and a secondary one to bet on it (Lopez-Gonzalez, Griffiths, et al., 2018), and (ii) sports betting advertising represents and promotes 
smartphones as the most normative devices to engage in online gambling (LopezGonzalez, Guerrero-Solé, et al., 2018).

\section{Conclusion}

The study here offers a novel perspective on the effects of smartphone technology on people trying to recover from gambling-related problems. The findings are grounded on data provided by individuals diagnosed with gambling disorder and whose preferred mode of play was smartphone gambling, a specialised subgroup relatively difficult to target. The study uncovered some of the singular characteristics of smartphone gambling and provided context to consider smartphones as essentially distinctive gambling devices, with attributes that differ not only from land-based gambling but from other forms of internet-based gambling in terms of intermittent but frequent reinforcement schedules that reflect the structural design of gambling products. Harm minimisation public health strategies must be cognizant of these singularities and adapt their approach to gambling prevention taking into consideration how gamblers interact with their smartphones.

In summary, the colonisation of non-gambling spheres by smartphone gambling proposed here has far-reaching implications for gambling disorder treatment practices in terms of reducing and controlling the exposure to gambling stimuli without interfering with individuals' privacy rights and their need for intimate spaces. Furthermore, changes in gambling disorder practice must be accompanied by a new understanding of gambling problems by both policymakers and stakeholders. This means adopting a broader definition of gambling that takes into account the whole spectrum of harms related to gambling and not solely the most immediate and visible consequences for individual gamblers.

\section{References}

Alimoradi, Z., Lin, C. Y., Broström, A., Bülow, P. H., Bajalan, Z., Griffiths, M. D., Ohayon, M. M., \& Pakpour, A. H. (2019). Internet addiction and sleep problems: A systematic review and meta-analysis. Sleep Medicine Reviews, 47, 51-61. https://doi.org/10.1016/j.smrv.2019.06.004

Ames, G. M., \& Grube, J. W. (1999). Alcohol availability and workplace drinking: Mixed method analyses. Journal of Studies on Alcohol, 60(3), 383-393. 
https://doi.org/10.15288/jsa.1999.60.383

Baggio, S., Dupuis, M., Berchtold, A., Spilka, S., Simon, O., \& Studer, J. (2017). Is gambling involvement a confounding variable for the relationship between internet gambling and gambling problem severity? Computers in Human Behavior, 71, 148152. https://doi.org/10.1016/j.chb.2017.02.004

Bergen, N., \& Labonté, R. (2019). "Everything is perfect, and we have no problems": Detecting and limiting social desirability bias in qualitative research. Qualitative Health Research. https://doi.org/10.1177/1049732319889354

Binde, P. (2016). Gambling-related embezzlement in the workplace: A qualitative study. International Gambling Studies, 16(3), 391-407. https://doi.org/10.1080/14459795.2016.1214165

Bouton, M. E., Woods, A. M., \& Todd, T. P. (2014). Separation of time-based and trialbased accounts of the partial reinforcement extinction effect. Behavioural Processes, 101(January), 23-31. https://doi.org/10.1016/j.beproc.2013.08.006

Braun, V., \& Clarke, V. (2006). Using thematic analysis in psychology. Qualitative Research in Psychology, 3, 77-101. https://doi.org/10.1191/1478088706qp063oa

Braverman, J., Laplante, D. A., Nelson, S. E., \& Shaffer, H. J. (2013). Using cross-game behavioral markers for early identification of high-risk internet gamblers. Psychology of Addictive Behaviors, 27(3), 868-877. https://doi.org/10.1037/a0032818

Browne, M., Greer, N., Rawat, V., \& Rockloff, M. (2017). A population-level metric for gambling-related harm. International Gambling Studies, 17(2), 163-175. https://doi.org/10.1080/14459795.2017.1304973

Chóliz, M., Marcos, M., \& Lázaro-Mateo, J. (2019). The risk of online gambling: A study of gambling disorder prevalence rates in Spain. International Journal of Mental Health and Addiction. https://doi.org/10.1007/s11469-019-00067-4

Deans, E. G., Thomas, S. L., Daube, M., \& Derevensky, J. (2017). The role of peer influences on the normalisation of sports wagering: A qualitative study of Australian men. Addiction Research \& Theory, 25(2), 103-113. https://doi.org/10.1080/16066359.2016.1205042

Dickerson, M., \& O'Connor, J. (2006). Gambling as an addictive behaviour. Cambridge University Press.

Drennan, J., Previte, J., Luck, E., \& Mort, G. S. (2018). M-Gambling - A strategic social marketing approach to protect vulnerable consumers. 
Estévez, A., Rodríguez, R., Díaz, N., Granero, R., Mestre-Bach, G., Steward, T., Fernández-Aranda, F., Aymamí, N., Gómez-peña, M., Del Pino-Gutiérrez, A., Baño, M., Moragas, L., Mallorquí-Bagué, N., López-González, H., Jauregui, P., Onaindia, J., Martín-Romera, V., Menchón, J. M., \& Jiménez-Murcia, S. (2017). How do online sports gambling disorder patients compare with land-based patients? Journal of Behavioral Addictions, 6(4). https://doi.org/10.1556/2006.6.2017.067

Fox, M., Philips, L., \& Vaidyanathan, G. (2003). Managing internet gambling in the workplace. First Monday, 8(4). https://doi.org/https://doi.org/10.5210/fm.v8i4.1044

Gainsbury, S. M., Angus, D. J., \& Blaszczynski, A. (2019). Isolating the impact of specific gambling activities and modes on problem gambling and psychological distress in internet gamblers. BMC Public Health, 19, 1372. https://doi.org/10.1186/s12889-019-7738-5

Gainsbury, S. M., Liu, Y., Russell, A. M. T., \& Teichert, T. (2016). Is all internet gambling equally problematic? Considering the relationship between mode of access and gambling problems. Computers in Human Behavior, 55(B), 717-728. https://doi.org/10.1016/j.chb.2015.10.006

Gainsbury, S. M., Russell, A., Wood, R., Hing, N., \& Blaszczynski, A. (2014). How risky is internet gambling? A comparison of subgroups of Internet gamblers based on problem gambling status. New Media \& Society, 17(6), 861-879. https://doi.org/10.1177/1461444813518185

Gambling Commission. (2020). Gambling participation in 2019: Behaviour, awareness and attitudes. https://www.gamblingcommission.gov.uk/PDF/surveydata/Gambling-participation-in-2019-behaviour-awareness-and-attitudes.pdf

González-Roz, A., Fernández-Hermida, J. R., Weidberg, S., Martínez-Loredo, V., \& Secades-Villa, R. (2017). Prevalence of problem gambling among adolescents: A comparison across modes of access, gambling activities, and levels of severity. Journal of Gambling Studies, 33(2), 371-382. https://doi.org/10.1007/s10899-0169652-4

Grant, J. E., Odlaug, B. L., \& Mooney, M. E. (2012). Telescoping phenomenon in pathological gambling. Journal of Nervous and Mental Disease, 200(11), 996-998. https://doi.org/10.1097/nmd.0b013e3182718a4d

Gray, H. M., Jónsson, G. K., LaPlante, D. A., \& Shaffer, H. J. (2015). Expanding the study of internet gambling behavior: Trends within the Icelandic lottery and sportsbetting platform. Journal of Gambling Studies, 31(2), 483-499. 
https://doi.org/10.1007/s10899-013-9427-0

Griffiths, M. D. (2009). Internet gambling in the workplace. Journal of Workplace Learning, 21(8), 658-670. https://doi.org/10.1108/13665620910996197

Griffiths, M. D., \& Parke, J. (2002). The social impact of Internet gambling. Social Science Computer Review. https://doi.org/10.1177/08939302020003008

Hartley, J. (2004). Case study research. In C. Cassell \& G. Symon (Eds.), Essential guide to qualitative methods in organizational research (pp. 323-333). Sage.

Hing, N., \& Gainsbury, S. (2013). Workplace risk and protective factors for gambling problems among gambling industry employees. Journal of Business Research, 66(9), 1667-1673. https://doi.org/10.1016/j.jbusres.2012.12.013

Hubert, P., \& Griffiths, M. D. (2018). A comparison of online versus offline gambling harm in Portuguese pathological gamblers: An empirical study. International Journal of Mental Health and Addiction, 16(5), 1219-1237. https://doi.org/10.1007/s11469-017-9846-8

James, R. J. E., O’Malley, C., \& Tunney, R. J. (2017). Understanding the psychology of mobile gambling: A behavioural synthesis. British Journal of Psychology, 108(3), $608-625$.

James, R. J. E., O’Malley, C., \& Tunney, R. J. (2019). Gambling on smartphones: A study of a potentially addictive behaviour in a naturalistic setting. European Addiction Research, 25(1), 30-40. https://doi.org/10.1159/000495663

Jiménez-Murcia, S., Alvarez-Moya, E. M., Stinchfield, R., Fernández-Aranda, F., Granero, R., Aymamí, N., Gómez-Peña, M., Jaurrieta, N., Bove, F., \& Menchón, J. M. (2010). Age of onset in pathological gambling: clinical, therapeutic and personality correlates. Journal of Gambling Studies, 26(2), 235-248. https://doi.org/10.1007/s10899-009-9175-3

Kohlbacher, F. (2006). The use of qualitative content analysis in case study research. Forum: Qualitative Social Research, 7(1), 21.

LaPlante, D., Schumann, A., LaBrie, R. A., \& Shaffer, H. J. (2008). Population trends in internet sports gambling. Computers in Human Behavior, 24(5), 2399-2414. https://doi.org/10.1016/j.chb.2008.02.015

Lloyd, J., Doll, H., Hawton, K., Dutton, W. H., Geddes, J. R., Goodwin, G. M., \& Rogers, R. D. (2010). Internet gamblers: A latent class analysis of their behaviours and health experiences. Journal of Gambling Studies, 26, 387-399. https://doi.org/10.1007/s10899-010-9188-y 
Lopez-Gonzalez, H., Griffiths, M. D., \& Estévez, A. (2018). In-play betting, sport broadcasts, and gambling severity: A survey study of Spanish sports bettors on the risks of betting on sport while watching It. Communication \& Sport. https://doi.org/10.1177/2167479518816338

Lopez-Gonzalez, H., Guerrero-Solé, F., \& Griffiths, M. D. (2018). A content analysis of how 'normal' sports betting behaviour is represented in gambling advertising. Addiction Research and Theory, 26(3), 238-247. https://doi.org/10.1080/16066359.2017.1353082

Mihaylova, T., Kairouz, S., \& Nadeau, L. (2013). Online poker gambling among university students: Risky endeavour or harmless pastime? Journal of Gambling Issues, 27, 15. https://doi.org/10.4309/jgi.2012.27.15

Myllymaa, A. (2017). The political economy of online gambling in the European Union [University of Helsinki]. https://helda.helsinki.fi/handle/10138/175678

Nower, L. (2003). Pathological gamblers in the workplace: A primer for employers. Employee Assistance Quarterly, 18(4), 55.

Papineau, E., Lacroix, G., Sévigny, S., Biron, J. F., Corneau-Tremblay, N., \& Lemétayer, F. (2018). Assessing the differential impacts of online, mixed, and offline gambling. International Gambling $\quad$ Studies, $\quad$ 18(1), 69-91. https://doi.org/10.1080/14459795.2017.1378362

Parke, A., \& Parke, J. (2019). Transformation of sports betting into a rapid and continuous gambling activity: a grounded theoretical investigation of problem sports betting in online settings. International Journal of Mental Health and Addiction. https://doi.org/10.1007/s11469-018-0049-8

Paul, R. J., \& Townsend, J. B. (1998). Managing workplace gambling-Some cautions and recommendations. Employee Responsibilities and Rights Journal, 11(3), 171186. https://doi.org/10.1023/A:1027355316884

Petry, N. M. (2016). Gambling Disorder: The first officially recognized behavioral addiction. In N. M. Petry (Ed.), Behavioral addictions: DSM-5® and beyond (pp. 7-42). Oxford University Press.

Saldaña, J. (2009). The coding manual for qualitative researchers. The coding manual for qualitative researchers. Sage.

Sheller, M., \& Urry, J. (2003). Mobile transformations of 'public' and 'private' life. Theory, Culture \& Society, 20(3), 107-125. https://doi.org/10.1177/02632764030203007 
Slutske, W. S. (2019). Genetic and environmental contributions to risk for disordered gambling. In Heinz, A., Romanczuk-Seiferth, N., \& Potenza, M. (Eds.), Gambling disorder (pp. 73-100). Springer, Cham. https://doi.org/10.1007/978-3-030-030605 5

Tolchard, B., \& Battersby, M. W. (2013). Cognitive behaviour therapy for problem gamblers: A clinical outcomes evaluation. Behaviour Change, 30(1), 12-23. https://doi.org/10.1017/bec.2013.2

Tong, A., Sainsbury, P., \& Craig, J. (2007). Consolidated criteria for reporting qualitative research (COREQ): A 32-item checklist for interviews and focus groups. International Journal for Quality in Health Care, 19(6), 349-357. https://doi.org/10.1093/intqhe/mzm042

Vandewater, C. (2013). Office betting survey 2013. Vault.com. https://www.vault.com/blogs/workplace-issues/office-betting-survey-2013-theresults-are-in

Wardle, H., Keily, R., Astbury, G., \& Reith, G. (2014). 'Risky places?': Mapping gambling machine density and socio-economic deprivation. Journal of Gambling Studies, 30(1), 201-212. https://doi.org/10.1007/s10899-012-9349-2

Wardle, H., Moody, A., Griffiths, M., Orford, J., \& Volberg, R. (2011). Defining the online gambler and patterns of behaviour integration: Evidence from the British Gambling Prevalence Survey 2010. International Gambling Studies, 11(3), 339356. https://doi.org/10.1080/14459795.2011.628684

Winnick, M., \& Zolna, R. (2016). Mobile touches: a study on how humans use technology. Dscout. https://blog.dscout.com/mobile-touches

Zakiniaeiz, Y., Cosgrove, K. P., Mazure, C. M., \& Potenza, M. N. (2017). Does telescoping exist in male and female gamblers? Does it matter? Frontiers in Psychology, 8, 1510. https://doi.org/10.3389/fpsyg.2017.01510

Zhao, Y., Marchica, L., Derevensky, J. L., \& Ivoska, W. (2018). Mobile gambling among youth: A warning sign for problem gambling? Journal of Gambling Issues, 38, 268282. https://doi.org/10.4309/jgi.2018.38.14 